\title{
A Pattern-Theoretic Characterization of Biological Growth
}

\author{
Ulf Grenander, Anuj Srivastava*, and Sanjay Saini
}

\begin{abstract}
Mathematical and statistical modeling of biological growth is an important problem in medical diagnostics. Here, we seek tools to analyze changes in anatomical parts using images collected over time. We introduce a structured model, called Growth by Random Iterated Diffeomorphisms (GRID), that treats a cumulative growth deformation as a composition of several elementary deformations. Each elementary deformation applies to a small region by capturing deformation local to that region and is characterized by a seed and a radial deformation pattern around that seed. These GRID variables-seed locations and radial deformation patterns-are estimated from observed images in two steps: 1) estimate a cumulative deformation over an observation interval; 2) estimate GRID variables using maximum-likelihood criterion from this estimated cumulative deformation. We demonstrate this framework using an MRI image data of a rat's brain growth. For future statistical analysis, we propose a time-varying Poisson process for the seed placements and a random drawing from a predetermined catalog of deformations for the radial deformation patterns.
\end{abstract}

Index Terms-Growth dynamics, growth models, growth patterns, random diffeomorphism.

\section{INTRODUCTION}

$\mathbf{M}$ ATHEMATICAL modeling and analysis of biological shape changes are of great interest in many branches of sciences, including physiology, ecology, evolution, medicine, and surgery. Medical applications include analysis and prediction of tumor growth in animal tissues, testing normality of an organ's growth, analysis of shapes of anatomical structures in brain, and tracking of biological growth and decay in body parts. Shapes and shape variations of anatomical parts are often important factors in deciding the state of health of a person. In many cases, the current clinical practice is to manually measure certain coarse characteristics such as lengths, sizes, or areas associated with the regions of interest, and use those indicators in diagnoses. Another common approach is to model objects via simple geometries, such as ellipsoids, spheres, and rectangles, and use the corresponding shape parameters for analysis. As an

Manuscript received September 29, 2006; revised December 11, 2006. This work was supported in part by the ARO under Grant W911NF-04-1-0268 and Grant W911NF-04-1-0113 and in part by the AFOSR under Grant FA9550-061-0324. Asterisk denotes corresponding author.

U. Grenander is with the Division of Applied Mathematics, Brown University, Province, RI 02912 USA.

*A. Srivastava is with the Department of Statistics, Florida State University, Tallahassee, FL 32306 USA (e-mail: anuj @ stat.fsu.edu).

S. Saini is with the Department of Statistics, Florida State University, Tallahassee, FL 32306 USA.

Color versions of one or more of the figures in this paper are available online at http://ieeexplore.ieee.org.

Digital Object Identifier 10.1109/TMI.2006.891500 example, for measuring the growth of a tumor some techniques use "multicellular spheroid" models, where the growth of the inner core of a tumor is modeled by elliptical spheroids [1]. In general, however, one would like to measure and analyze complete shapes of objects and not just certain indicators or their approximations to simple geometries. In this paper, we develop a mathematical framework for analyzing growth of biological objects such as animal cells, anatomical parts, tumors, and organs.

\section{A. Comparison With Past Work}

Three broad categories of past papers are relevant for comparison here. These categories are: shape analysis using anatomical landmarks, shape modeling and comparisons using background deformations, and explicit mathematical models for growth of tumors.

The subject of growth and development has formally been studied for several decades. Many current techniques in statistical shape analysis [2]-[4] use a collection of predetermined anatomical landmarks (salient points on objects) to analyze shape changes. In contrast, we will focus on analyzing full images of complete shapes, including interiors and boundaries. Notably, we propose to use both the pixel values and pixel locations, instead of using just the locations of landmarks in the images.

Growth and shape dissimilarities have also been modeled in the past using smooth deformations, or diffeomorphisms, of the underlying coordinate systems [5]-[11]. The set of all diffeomorphisms from $\mathbb{R}^{n}$ to $\mathbb{R}^{n}$, with $n=2$ or 3 , denoted by $\mathcal{D}$, forms an infinite-dimensional space, and its differential geometry [12] is often used to analyze deformations. An element $\phi \in \mathcal{D}$ denotes a diffeomorphism such that a pixel located at $x \in \mathbb{R}^{n}$ is mapped to the location $\phi(x) \in \mathbb{R}^{n}$. We are interested in a subset of $\mathcal{D}$ that is naturally suited to modeling biological deformations. While a large extent of transformations in $\mathcal{D}$ maybe relevant in studying shape dissimilarities across subjects, or across classes/species, a smaller, more structured subset seems appropriate for studying growth of an anatomical object, at least over small time intervals. In case of biological growths, it seems more natural to model a time-sequence of smaller, more elementary deformations, rather than a large integrated deformation, to highlight the time-varying nature of growth.

Lastly, several groups have developed explicit models for capturing the growth of tumors as seen in images. These models utilize mathematical tools such as reaction-diffusion equations [13], mechanical models [13], or cellular automation [14], and provide a remarkable insight into the growth process. The paper by Swanson $e t$ al. [15] and the book by Chaplain [16] describe several of the existing models used in studying tumor growth. 
We seek a growth model capable of modeling growth in a variety of anatomical parts, including tumors. Our approach can lead to a stochastic differential equation modeling local deformations, similar to the diffusion model of [13], but our framework allows for a more efficient representation of a wider class of deformation patterns. Ling and He [17] have studied certain biological growth models using entropy considerations. Our approach is quite similar to the local transformations introduced in Arsigny et al. [10] except that they restrict to a smaller set of rigid or affine transformations.

\section{B. Issues in Representation of Growth Patterns}

A fundamental question in computational anatomy is: How can we construct a family of mappings for representing biological variations and growth? Furthermore, what dynamical models can be imposed on these mappings to characterize typical biological growths? We shall introduce a type of random diffeomorphism to prepare for the statistical analysis of biological growth patterns. A related question that is addressed in this paper is: What coordinate system(s) should be used to analyze biological growth? Instead of a fixed cartesian or a polar coordinate system, it is desirable to have an evolving coordinate representation that reflects changing anatomy of object(s) under study and that facilitates biologically plausible models. Our goal is to develop representations that isolate and focus on deformations of biological interest and result in a more efficient and tractable analysis.

\section{Our Approach}

We propose a Growth (by) Random Iterated Diffeomorphisms (GRID) model to impose a structure on the desired family of deformations. In this model, an overall growth is modeled by a sequence of small, local deformations, each being a diffeomorphism somewhat closer to identity transformation $i d \in \mathcal{D}$. Their individual effects maybe small but their cumulative effects are significant. Each elementary diffeomorphism shall be primarily concentrated around a focal point of growth, called a seed. A small active area surrounding the seed either grows or decays, or both, radially according to a radial deformation pattern. This pattern is specified in a polar coordinate system centered at the seed using two functions-angular and radial deformation functions (ADFs and RDFs). These functions can often be further simplified using parametric descriptions. Areas beyond the region of active growth are pushed in or out, according to the changes in the inner active area, or simply remain unchanged.

The choice of GRID model is motivated by observations of locally active growths in several biological systems. Changes in objects are seldom homogeneous and are characterized by heterogeneities that result from varying biochemical compositions of that region. For example, certain proteins act as catalysts for tissue growth, and their concentration levels dictate the scale and the nature of resulting growths. Higher concentrations trigger active growth (or decay) in their neighborhoods. As another example, in case of a single cell, the growth may be caused by activation of some genes over a period, while the other genes remain passive during that same period. Different genes may activate at different times, and the nature of resulting deformations

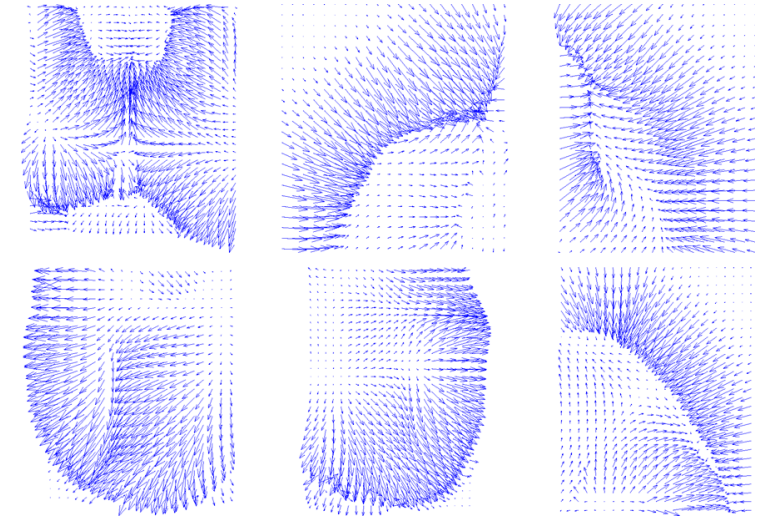

Fig. 1. Examples of growth-related deformation fields that motivate use of radial deformation fields around imaginary focal points called seeds.

may differ as well. Incorporation of a locally active deformation is an important highlight of the GRID model.

The choice of radial nature of deformation is motivated by observed growth patterns. Shown in Fig. 1 are some examples of actual deformation fields in high-growth subregions, estimated from MRI images of a rat's brain. As these pictures suggest, it is possible to efficiently characterize these deformations as radial vector fields, emanating from some imaginary focal points. Some advantages of the GRID model, over current methods that estimate full deformation, are as follows.

1) Biologically Plausible Representations: An important aspect of this model is its ability to represent and analyze growth/decay locally. The use of seeds and radial growth patterns seems to capture well the deformations induced by observed biological growth, in our limited experiments so far. A large concentration of seeds in a region implies high levels of active biochemical ingredients in that region. Similarly, types of ADFs point to the nature of these catalysts: growth-oriented, decay-oriented, or a composite. In contrast, it is harder to ascribe such biological interpretations to cumulative deformations that are estimated in current methods.

2) Parametric Nature of Representations: The proposed model incorporates a rich family of patterns, accounting for different combinations of growths, decays, or others. The resulting deformations can be analyzed using seed locations and deformation functions (DFs). It provides an efficient framework for representing and analyzing growth, using these model variables. Contrast this with a nonparametric approach where the full deformations are to be analyzed to characterize growth. We do, however, use full deformations to arrive at the model variables in this paper. In principle, one should be able to analyze growth by estimating the GRID variables directly from the image data. That is a long-term goal to be explored in our future work. The parameters generating GRID deformations should most naturally be treated as random values with appropriate prior probabilities. However, the experiments presented later in this paper consider these parameters as unknown, deterministic quantities leading to a maximum-likelihood estimation (MLE) framework. 
The choice of prior models and the resulting maximum a posteriori (MAP) estimation is briefly outlined under future extensions.

3) Statistical Modeling of Growth: Another important consequence of this model is the potential statistical framework it will provide to detect, classify, and recognize growth patterns. Statistical models on seed placements and random choices of deformations functions can provide randomness of growth models. For instance, one can characterize growth patterns using: 1) intensity functions associated with Poisson seed placements; 2) the nature of observed DFs. Contrast this with a statistical analysis on full space $\mathcal{D}$ without any additional structure. The models on seed placements and DFs can be also used to induce explicit probability models on the deformation fields.

In this paper, the estimation of GRID variables is performed in two steps. First, we estimate a cumulative deformation map representing biological growth during a time period. Then, we estimate GRID variables—-seed placements and DFs—-from this deformation map.

The rest of this paper is organized as follows. We introduce the GRID model—seed placements and radial deformation functions-in Section II. Some interesting analytical properties associated with the deformation field generated by the GRID model are presented in Section III. Estimation of diffeomorphic maps, using time-sequences of images, is explained in Section IV, while the use of these maps to estimate MLEs of GRID variables is illustrated in Section V. We conclude the paper with a listing of possible future extensions in Section VI and with a summary in Section VII.

\section{GRID MODEL}

A starting point for modeling biological growth is at the cellular level. Studies of cellular growth are motivated in part by Omne vivum ex ovo-all life from egg-a famous statement by William Harvey [18]. Motivated by pre-eminent role of cellular divisions in all anatomical growth, we model growth using a sequence of local elementary deformations. We shall indeed start from an initial shape taking up the compact region. For simplicity, we shall start assuming growth to take place in $\mathbb{R}^{2}$; much can be carried over to $\mathbb{R}^{3}$ with obvious modifications. We will denote the growing region at time $t$ by $X(t) \subset \mathbb{R}^{2}$. In case of MRI images, $X(t)$ is a subset of imaged coordinates expressed in a Cartesian system.

\section{A. Iterated Diffeomorphisms}

We assume that a deformation induced by growth consists of several elementary deformations. These elementary deformations are assumed to result from random placements of growth centers, called seeds, and a random choice of DFs to model growth radially around those seeds. At time $t$, let the elementary growth result in a diffeomorphism $\phi_{t}$ such that $\phi_{t}: X(t) \mapsto$ $X(t+1)$. The point represented by $x(t)$ moves to $x(t+1)=$ $\phi_{t}(x(t))$ under this transformation. We seek a biologically motivated representation of $\phi_{t}$ as follows.

1) Deformation Seed: We hypothesize that the deformation $\phi_{t}$ is local in the sense that it is concentrated around a central point, or a seed, denoted by $x_{\text {seed }}^{t} \in X(t)$. The seed $x_{\text {seed }}^{t}$ denotes an activated point while the other points may remain passive at $t$. For statistical modeling, one can assume that the seeds are distributed according to a Poisson process and the underlying intensity function reflects the concentration of active biochemical ingredients.

2) Deformation Type: Once a seed is placed, the deformation in a neighborhood around it is modeled using radial vector fields, which in turn are generated by DFs mentioned earlier. The actual choice of DFs is based on discrete decisions of cells as controlled by genes that are switched on at the moment. This could be, e.g. mitosis, corresponding to proper growth, or cell death meaning decay. Momentarily, we use $\alpha$ to represent the DFs; it dictates whether a deformation around a seed results in growth, decay, or a mixture, and the specific pattern associated with that deformation. We conjecture that the set of deformations that occur frequently in nature is a finite set and, therefore, one can view $\alpha$ as an index to this set.

Note that $\phi_{t}$ depends on both the seed $x_{\text {seed }}^{t} \in X(t)$ and the deformation type $\alpha$. The interesting part of $\phi_{t}$ lies inside an active region containing the seed; outside this region the coordinates remain unchanged. A sequence of events at times $t_{1}, t_{2}, \ldots t_{n}$, at the corresponding seed locations $x_{\text {seed }}^{1}, x_{\text {seed }}^{2}, \ldots x_{\text {seed }}^{n}$, and the deformation types $\alpha_{1}, \alpha_{2}, . ., \alpha_{n}$, leads to an iterated diffeomorphism

$$
\Phi_{t_{1}}^{t_{n}} \equiv \phi_{t_{n}} \circ \ldots \circ \phi_{t_{2}} \circ \phi_{t_{1}}
$$

where $\phi_{t_{i}}$ is applied to the resulting $x$-field $X\left(t_{i-1}\right)$. In view of the random placements of seeds, and the random nature of the diffeomorphisms, characterized by $\alpha$ s, the growth will be random in nature. This is the GRID model, in its general form. We shall gradually specialize it in the next few sections.

\section{B. Elementary Translation-Invariant Deformation}

Here, we start by developing an elementary transformation $\phi_{t}$. We shall concentrate our study to situations where the diffeomorphism $\phi_{t}$ is invariant with respect to the translation group in $\mathbb{R}^{2}$. In other words, there exists a diffeomorphism $\psi_{t}: \mathbb{R}^{2} \mapsto \mathbb{R}^{2}$ such that

$$
\phi_{t}(x)=x_{\text {seed }}^{t}+\psi_{t}\left(x-x_{\text {seed }}^{t}\right) .
$$

Let $d \phi_{t}(x)$ denote the cumulative change in the position of $x$ over the interval $[t, t+1]$. It is given by $d \phi_{t}(x)=\phi_{t}(x)-x=$ $\psi_{t}\left(x-x_{\text {seed }}^{t}\right)-\left(x-x_{\text {seed }}^{t}\right)$. Defining a new diffeomorphic map, $\theta_{t}: \mathbb{R}^{2} \mapsto \mathbb{R}^{2}$ such that $\theta_{t}(u)=\psi_{t}(u)-u$. We can rewrite the displacement vector as

$$
d \phi_{t}(x)=\theta_{t}\left(x-x_{\text {seed }}^{t}\right) .
$$

The displacement of a point $x$ at a time $t$ is dependent only on its location relative to the seed at that time and the nature of $\theta_{t}$. Note that different points can act as seeds at different times. This setup is illustrated in the left panel of Fig. 2, which shows a seed, indicated by $x_{\text {seed }}$, and a location, indicated by $x$, being considered for growth, all for a fixed $t$. We have introduced three ways of specifying the mapping that takes $x(t)$ to $x(t+1)$. 


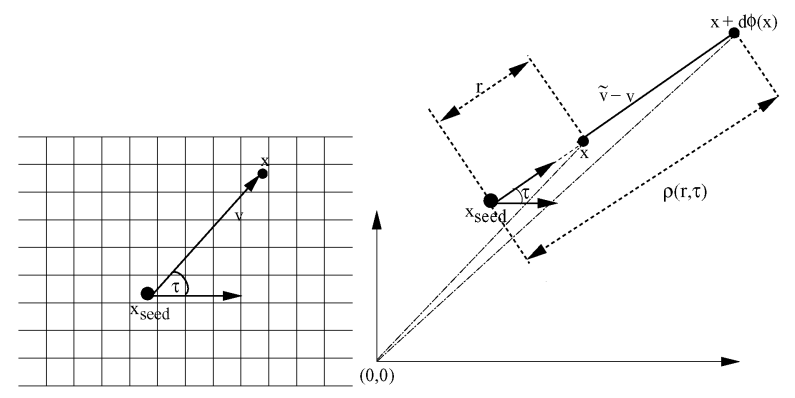

Fig. 2. Left: deformation coordinates for a point $x$ relative to the seed. Right: illustration of displacement of $x$ to $x+d \phi_{t}(x)$ using a radial growth function that takes $v=(r, \tau)$ to $\tilde{v}=(\rho(r, \tau), \tau)$.

1) $\phi_{t}$ provides the new location of the point in the fixed, absolute cartesian coordinate system in $\mathbb{R}^{2}$ centered at the origin.

2) $\psi_{t}$ provides the new location of a genetic material in a cartesian coordinate system that is centered at $x_{\text {seed }}^{t}$. Very often we will use polar coordinates of points in this system as follows: the point $v=x-x_{\text {seed }}^{t}$ in this system has polar coordinates $(r, \tau)$ such that $v=[r \cos (\tau), r \sin (\tau)]^{T}$. As a convention, the angle $\tau$ will be measured anticlockwise from the positive, horizontal axis.

3) $\theta_{t}$ computes the displacement vector directly in a fixed cartesian coordinate system. Later in Section V-B, we impose an observation model on the displacement field to setup a maximum likelihood estimation problem.

We will use these three mappings interchangeably in our discussion depending on the convenience.

The restriction to translation invariant deformations still leaves a lot of leeway in defining these mappings. We further constrain the deformation $\psi_{t}$ to be radial as follows. Let $(r, \tau)$ be the polar coordinates of the vector $v=x-x_{\text {seed }}^{t}$. In these local polar coordinates, the deformation $\psi_{t}$ is given by $(r, \tau) \mapsto(\rho(r, \tau), \tau)$, where the choice of $\rho(r, \tau)$ varies according to the model. This is pictorially illustrated in the right panel of Fig. 2. We will study a few examples of the radial displacement $\rho(r, \tau)$, each of which involves decomposing $\rho(r, \tau)$ into two functions, one for $r$ and other for $\tau$.

1) As a first example, let the scale change along $\tau$ direction be given by $\rho(r, \tau)=r+R(r) A(\tau)$, where $A: \mathbb{S}^{1} \mapsto \mathbb{R}$ is called the angular deformation function (ADF) and $R: \mathbb{R}_{+} \mapsto \mathbb{R}_{+}$is called the radial deformation function (RDF). The active area along the direction $\tau$ is expanded (or contracted) at the rate given by $A(\tau): A(\tau)>0$ which means expansion or growth while $A(\tau)<0$ means contraction or decay in that direction. Each of these two functions can either be used in a parametric or a nonparametric form. Based on experimental studies, we have chosen to represent RDF in a convenient parametric form while ADF is left in a functional form. There are several possibilities for the RDF $R$.

a) $R(r)=r \exp \left(-(r / c)^{2}\right)$, for $r \geq 0, c>0$.

b) $R(r)=(r / c)^{p-1} \exp (-(r / c))$, for $r \geq 0, p>0$, and $c>0$.

We will use $\delta$ to denote the parameters of $R$ and we will write $R(r)$ as $R(r ; \delta)$ when needed. In the first case $\delta=c$,

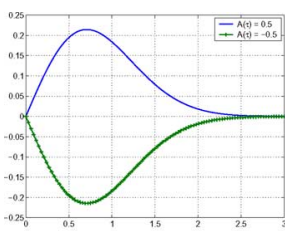

(a)

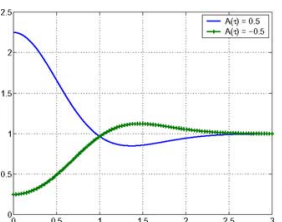

(b)

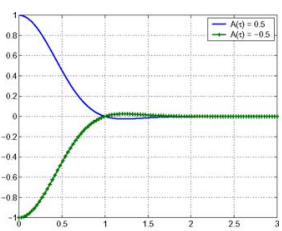

(c)
Fig. 3. (a) Examples of radial displacements $(\rho(r, \tau)-r)$ for $c=1, R(r)=$ $r e^{-r^{2} / c^{2}}$, and $A(\tau)=0,5,-0.5$. Plots of (b) determinant of the Jacobian and (c) divergence for the corresponding $\phi_{t}$.

while in the second case $\delta=(p, c)$. Plotted in Fig. 3(a) are two examples of $\rho(r, \tau)-r$ for $A(\tau)=-0.5$ and 0.5 for the case where $R(r)=r \exp \left(-(r / c)^{2}\right)$. (For a better understanding, this figure plots the radial displacement $\rho(r, \tau)-r$, rather than $\rho(r, \tau)$ itself.) As the two curves show, major deformation lies in a region around $x_{\text {seed }}^{t}$, denoted by $r=0$, here, and goes to zero exponentially fast as $r$ gets larger.

2) Another possibility is to define $\rho(r, \tau)$ according to

$$
\rho(r, \tau)=\left\{\begin{array}{ll}
A(\tau) r, & \text { if } r<c \\
(A(\tau)-1) c+r, & \text { if } r \geq c
\end{array} .\right.
$$

As earlier, $A(\tau)$ is the ADF. In each direction, $\rho(r, \tau)$ is piecewise linear in $r . \rho(r, \tau)$ is scaled up or down according to $A(\tau)$, in the active region. Here, $A(\tau)>1$ means expansion while $A(\tau)<1$ means contraction. The main difference here, from the previous model, is that here the outer regions are also transformed; they are pushed away (or inwards) according to the expansion (or contraction) in the inner active region. This model is not explored further in this paper.

Through these choices of $\rho(r, \tau)$, we have ensured that the interesting part of the deformation $\phi_{t}$ takes place inside a neighborhood of $x_{\text {seed }}^{t}$, a subset of $X(t)$, whose size depends on $\delta$. In the first case, the outside regions are unchanged, while in second the outside regions are simply pushed in or out. In the coordinate system centered at $x_{\text {seed }}^{t}$, the point located at $v \equiv(r, \tau)$ moves to the location $\tilde{v} \equiv \rho(r, \tau) u(\tau)$, where $u(\tau)=[\cos (\tau), \sin (\tau)]^{T}$ is a unit vector. The three mappings introduced earlier can be restated in this notation as follows: $\psi_{t}(v)=\tilde{v}, \theta_{t}(v)=\psi_{t}(v)-v$, and $\phi_{t}\left(x_{\text {seed }}^{t}+v\right)=x_{\text {seed }}^{t}+\tilde{v}$

\section{Examples of ADFs}

To illustrate elementary deformations resulting from GRID model, we show some examples representing the primitive forms of growth/decay. For brevity, we will restrict to radial growth characterized by $R(r)=r \exp \left(-(r / c)^{2}\right)$, for a certain $c$. As mentioned earlier, a rich family of deformations can be obtained by simply changing the ADF. The behavior is regulated mainly by the maxima and minima of the ADF $A$. If $A$ has a single extremum, we label it the unipolar growth; if it has two extrema we call it the bipolar growth, and the case with several minima and maxima we call the multipolar growth. If the function $A$ is mostly positive, then we will label the corresponding seed as a source; if it is mostly negative, then we will call the seed a sink. In case $A$ is both positive and negative, then we have a composite seed. 


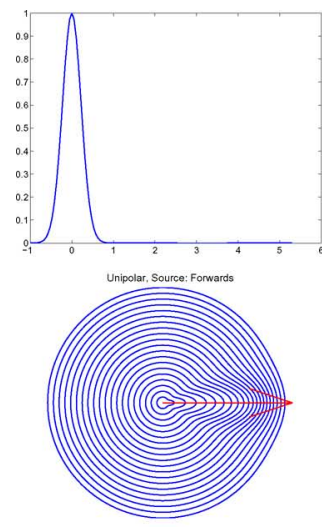

(a)

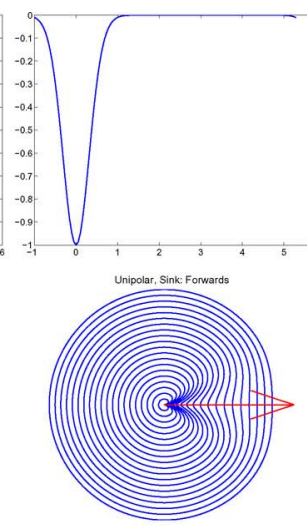

(b)

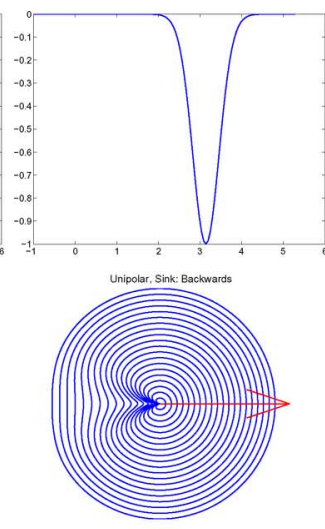

(c)

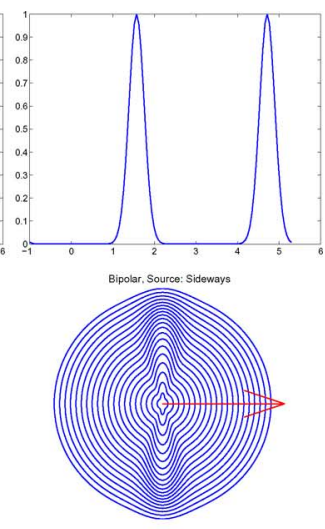

(d)

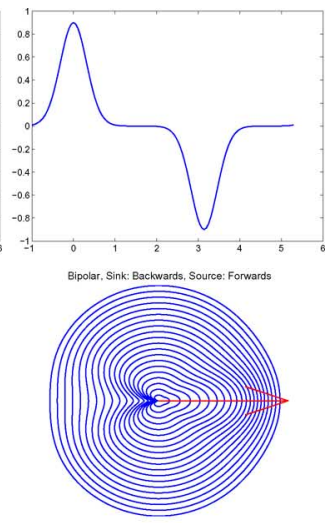

(e)

Fig. 4. Examples of elementary deformations $\psi_{t}$ obtained using different ADFs. (a)-(c) show unipolar ADFs and (d) and (e) show bipolar ADFs.
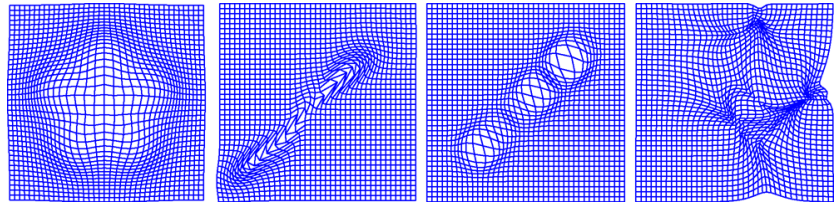

Fig. 5. Some examples of deformations resulting from random combinations of these elementary deformations.

Fig. 4 shows some examples of these elementary deformations $\phi_{t}$ applied to a uniform polar grid. For each case, we display the function $A(\tau)$ versus $\tau$ (top panel), with $\tau$ plotted in radians, and the resulting $\phi_{t}$ applied to a uniform polar grid (bottom panel). Shown in Fig. 4(a) is an example of unipolar source where there is a proper growth in the positive horizontal direction. The pattern shown in Fig. 4(d) is termed bipolar source as it results in a proper growth in two different directions. Next, consider a decay, so that the seed acts like a sink. Fig. 4(b) and (c) displays the case of decay in positive and negative horizontal directions. We term these cases unipolar sink. A further possibility is the bipolar behavior where the deformation results in growth in some direction and decay in some other direction. Shown in (4e) is the case of a source in one direction and a sink in another direction. One can construct a whole catalog of ADFs by choosing source or sink or composite, and polarity, and directions: $\{$ source, sink, composite $\}$ $\times\{$ unipolar, bipolar, multipolar $\} \times\left\{\mathbb{S}^{1} \times \mathbb{S}^{1} \ldots\right\}$. Under the GRID model one can characterize cumulative biological growth using a collection of seed locations and elementary deformations. Shown in Fig. 5 are some examples of cumulative deformations generated by a concatenation of these elementary deformations.

\section{ANALYSIS OF GRID MODEL}

In this section, we study certain analytical properties of deformations generated by the GRID model. We start by deriving conditions under which $\phi_{t}$ is a diffemorphism, followed by a study of Jacobian, divergence, and curl associated with GRIDgenerated deformation fields. Here, we consider the RDF to be $R(r)=r \exp \left(-(r / c)^{2}\right)$, but a similar analysis results for other choices of RDF.

\section{A. Diffeomorphic Nature of Mappings}

We seek conditions that ensure that the mappings $\phi_{t}$ are diffeomorphic at all times. Consider the displacement of $x$ under the mapping $\phi_{t}$. For the vector $v=x-x_{\text {seed }}^{t}$, and its polar representation $v=(r, \tau)$, the new relative location of this point is given by $\psi_{t}(v)=\tilde{v}$ whose polar coordinates are $(\rho(r, \tau), \tau)$. The mapping $\phi_{t}$ will be diffeomorphic as long as $\rho(\cdot, \tau): \mathbb{R}_{+} \mapsto$ $\mathbb{R}_{+}$is a diffeomorphism, for all $\tau$. So the next question is: When is $\rho$ a diffeomorphic function? Fix an angle $\tau$ and that fixes the value of $A(\tau) \equiv A$. Now, $\rho(\cdot, \tau)$ is a diffemorphism as long it has a piecewise-continuous derivative and $(\partial \rho / \partial r)>0$.

For the case $R(r)=r \exp \left(-(r / c)^{2}\right)$, we get $(\partial \rho / \partial r)=$ $1+A \exp (-w)(1-2 w)$, with $w=\left(r^{2} / c^{2}\right) \in \mathbb{R}_{+}$. From $(\partial \rho / \partial r)>0$, we obtain the condition that $1+A \exp (-w)(1-$ $2 w)>0$. The following cases result.

Case 1: For the value of $w=0$, the partial derivative is positive for all values of $A$.

Case 2: For $w<0.5$, this condition becomes $A>$ $(-\exp (w) /(1-2 w))$. Since the maximum value of the right side is -1 , achieved at $w=0$, we reach the condition that $A>-1$.

Case 3: For $w>0.5$, the condition becomes $A<$ $(-\exp (w) /(1-2 w))$. The minimum value of the right side is $\exp (1.5) / 2 \approx 2.2408$, achieved at $w=1.5$. Therefore, we need $A<2.2408$ for $\rho$ to be a diffeomorphism.

In summary, $\rho$ and $\phi_{t}$ are diffeomorphisms as long as $-1<$ $A<2.2408$ for all $\tau$. Recall from earlier that $A<0$ results in compression and $A>0$ results in expansion. The reader can check that all ADFs shown in Fig. 4 satisfy these conditions.

\section{B. Jacobian of Elementary GRID Flow}

To analyze $\phi_{t}$ further, one can compute its Jacobian. In physical terms, the Jacobian of $\phi_{t}$ at $x$ measures the change of area in a small region around $x$. Under the GRID model, this transformation is actually performed in polar coordinates, via a sequence of transformations given by

$$
\left(x_{1}, x_{2}\right) \stackrel{J_{1}}{\longrightarrow}(r, \tau) \stackrel{J_{2}}{\longrightarrow}(\rho(r, \tau), \tau) \stackrel{J_{3}}{\longrightarrow}\left(\tilde{x}_{1}, \tilde{x}_{2}\right)
$$


where $J_{1}, J_{2}$, and $J_{3}$ denote Jacobians of the three transformations. These Jacobians are given by

$$
\begin{aligned}
J_{1} & =\left(\begin{array}{cc}
\cos (\tau) & \sin (\tau) \\
-\frac{\sin (\tau)}{r} & \frac{\cos (\tau)}{r}
\end{array}\right), \quad J_{2}=\left(\begin{array}{cc}
\frac{\partial \rho}{\partial r} & \frac{\partial \rho}{\partial \tau} \\
0 & 1
\end{array}\right) \\
J_{3} & =\left(\begin{array}{cc}
\cos (\tau) & -\rho \sin (\tau) \\
\sin (\tau) & \rho \cos (\tau)
\end{array}\right)
\end{aligned}
$$

where $\rho$ is a short form of $\rho(r, \tau)$. The determinant of overall Jacobian is $\operatorname{det}\left(J_{1} J_{2} J_{3}\right)=\prod_{i=1}^{3} \operatorname{det}\left(J_{i}\right)=$ $(1 / r) \rho(r, \tau)(\partial \rho / \partial r)$. If $R(r)=r \exp \left(-(r / c)^{2}\right)$, this determinant is given by $\operatorname{det}\left(J_{1} J_{2} J_{3}\right)=\left(1+A(\tau) \exp \left(-r^{2} / c^{2}\right)\right)(1+$ $\left.A(\tau) \exp \left(-r^{2} / c^{2}\right)\left(1-2\left(r^{2} / c^{2}\right)\right)\right)$. Shown in Fig. 3(b) are two example plots of $\operatorname{det}\left(J_{1} J_{2} J_{3}\right)$, for $A(\tau)=-0.5,0.5$, under this model. At the seed $x_{\text {seed }}$, the determinant is given by $(1+A(\tau))^{2}$, while for points far away from the seed the determinant is one. For negative values of $A(\tau)$, the determinant is less than one, implying a reduction in area. Similarly, a positive $A(\tau)$ implies an increase in the area or growth around the seed. Since the determinant measures the change of area around a point, this result again highlights the locally active nature of the elementary GRID deformations.

\section{Fluid Characterization of GRID Flows}

It is possible to characterize in general terms the flows generated by the GRID model. Using (5), the full Jacobian for transformation $\phi_{t}$ from $\left(x_{1}, x_{2}\right)$ to $\left(\tilde{x}_{1}, \tilde{x}_{2}\right)$ is given by $M=J_{3} J_{2} J_{1}$ where

$$
\begin{aligned}
& M_{11}=\rho_{r} \cos ^{2}(\tau)-\frac{\rho_{\tau}}{r} \sin (\tau) \cos (\tau)+\frac{\rho}{r} \sin ^{2}(\tau) \\
& M_{12}=\rho_{r} \cos (\tau) \sin (\tau)+\frac{\rho_{\tau}}{r} \cos ^{2}(\tau)-\frac{\rho}{r} \sin (\tau) \cos (\tau) \\
& M_{21}=\rho_{r} \cos (\tau) \sin (\tau)-\frac{\rho_{\tau}}{r} \sin ^{2}(\tau)-\frac{\rho}{r} \sin (\tau) \cos (\tau) \\
& M_{22}=\rho_{r} \sin ^{2}(\tau)-\frac{\rho_{\tau}}{r} \sin (\tau) \cos (\tau)+\frac{\rho}{r} \cos ^{2}(\tau) .
\end{aligned}
$$

The divergence of the displacement vector field at a point is given by $\left(\partial \tilde{x}_{1} / \partial x_{1}\right)+\left(\partial \tilde{x}_{2} / \partial x_{2}\right)-2$, and it measures the rate at which biological matter (considered as fluid) expands in a unit region centered at that point. In case this divergence is zero for all points, the fluid is said to be incompressible. Similarly, the curl is given by $\left(\partial \tilde{x}_{1} / \partial x_{2}\right)-\left(\partial \tilde{x}_{2} / \partial x_{1}\right)$, and it measures the angular momentum, or rotational effect, of fluid particles flowing along streamlines of the given vector field. In case the curl of a vector field is zero at all points, the field is termed irrotational. In that case, the vector field can be written as the gradient of a potential function, i.e. there exists an $H$ such that $d \phi_{t}=\nabla H$. For biological growth, resulting from irrotational fields, one can use $H$ to interpret growth.

Substituting for the entries from the full Jacobian, we obtain the formulas for divergence and curl of $\psi_{t}$ under the GRID model.

1) The divergence is given by $\operatorname{div}\left(\phi_{t}\right)=(\partial \rho / \partial r)+(\rho / r)-2$.

2) The curl is given by $\operatorname{curl}\left(\phi_{t}\right)=(1 / r)(\partial \rho / \partial \tau)$.

In the case of $R(r)=r \exp \left(-(r / c)^{2}\right)$, the divergence of the deformation field at $(r, \tau)$ is given by $\operatorname{div}\left(\psi_{t}\right)=$ $2 A(\tau) \exp \left(-2 r^{2} / c^{2}\right)\left(1-r^{2} / c^{2}\right)$, while the curl is given by $\operatorname{curl}\left(\phi_{t}\right)=A^{\prime}(\tau) \exp \left(-r^{2} / c^{2}\right)$. Inside the active region, the divergence depends on the $\mathrm{ADF} A(\tau)$ : a positive value of $A(\tau)$ implies fluid expansion while a negative value implies contraction. Note that the influence of a seed goes down exponentially as we move far away from the seed. Similarly, the value of $A^{\prime}(\tau)$ dictates the curl inside the active region. For points outside the active region, both the divergence and the curl go to zero exponentially fast. An irrotational field is obtained when the ADF is isotropic, i.e. $A^{\prime}(\tau)=0$.

So far, we have introduced the GRID model and have presented some analytical properties of deformations resulting from this model. Next, we consider the problem of estimating model variables from raw image data.

\section{ESTIMATION OF GROWTH DEFORMATION}

In the next two sections, we present some results on estimation of GRID variables from MRI growth data of a rat's brain. This estimation is accomplished in two steps. First, we estimate the deformation $\phi_{t}$ that models brain growth from time $t$ to $t+1$. Later, we estimate seed locations, RDFs, and ADFs from the estimated $\phi_{t}$. This two-step approach has a limitation that the values of estimated GRID variables depend on the estimated $\phi_{t}$ which can be different under different criteria. A better approach in the future will be to estimate GRID variables directly from the image data, bypassing the estimation of $\phi_{t}$.

In this section, we describe the first step of estimating $\phi_{t}$ from the given image data at times $t$ and $t+1$. Let $I$ be the image at time $t$ and $J$ be the image at time $t+1$; we will simplify notation by fixing $t$ and dropping it from the subscript in this section. There is a large body of literature on estimation of deformations that model variations between given images. An important distinguishing factor between various techniques is the choice of cost function used in estimation of deformation. Ideas such as elastic matching [9], viscous modeling [19], spline-fitting [20], point-based matching [21], curve-based matching [22], and surface matching have been used in the past.

We perform this estimation using an energy that seeks to match both the images pixels and their boundaries. This energy that has three components: 1) $E_{1}$ reflects the squared error between the deformed image and the target images; 2) $E_{2}$ measures the mismatch between the (image) gradient vectors in the two images; 3) $E_{3}$ measures the smoothness of $\phi$. The total energy function is $E[\phi]=E_{1}+E_{2}+E_{3}$

$$
\begin{array}{r}
c_{1} \int_{\mathbb{R}^{2}}[I(\phi(x))-J(x)]^{2} d x+c_{2} \int_{\mathbb{R}^{2}} f(\langle\nabla I(\phi(x)), \nabla J(x)\rangle) d x \\
+c_{3} \int_{\mathbb{R}^{2}}\|\nabla(\phi(x)-x)\|^{2} d x \quad(6)
\end{array}
$$

with $f(y)=-\tanh (y / a) ; a>0$ and $c_{1}, c_{2}, c_{3}$ are positive constants that denote relative weights of the three components. We want to solve for $\hat{\phi}=\operatorname{argmin}_{\phi \in \mathcal{D}} E[\phi]$. Some important aspects of our implementation are as follows.

1) Image Interpolation: We will use an iterative gradient approach to solve for $\hat{\phi}=\operatorname{argmin}_{\phi} E[\phi]$. We need several gradients, for example $\partial E / \partial \phi$ on a discrete lattice. Since $\phi$ takes values in $\mathbb{R}^{2}$, the value of $I(\phi(x))$ may not be readily available as $I$ is defined only on the lattice. We will use the bilinear interpolation to estimate image values between lattice points to obtain $I(\phi(x))$. 


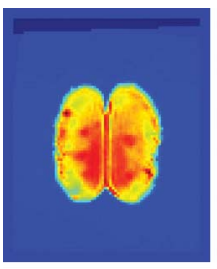

(a)

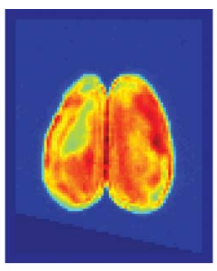

(b)

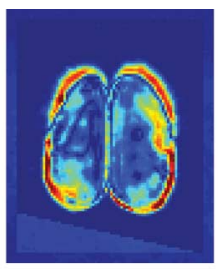

(c)

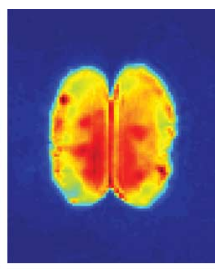

(d)
Fig. 6. (a) $I$, (b) $J$, (c) initial difference $I-J$, and (d) $I$ after RST.

2) Multiresolution Estimation: Let $h_{\sigma}$ be a 2-D Gaussian filter with a bandwith $\sigma$, and let $I_{\sigma}=I * h_{\sigma}$ and $J_{\sigma}=$ $J * h_{\sigma}$ be the smoothed versions of these two images. Define $\hat{\phi}(\sigma)=\operatorname{argmin}_{\phi} E[\phi, \sigma]$, where $E[\phi, \sigma]$ is as defined in (6), except $I$ and $J$ are replaced by $I_{\sigma}$ and $J_{\sigma}$, respectively. Our strategy is to start with a large $\sigma$, implying a coarse resolution to estimate the mapping $\phi$. In the next iteration, decrease $\sigma$, thus increasing the resolution, and use the previous estimate of $\phi$ to initialize the gradient search for $\phi$ at the next resolution.

3) Rotation, Scaling, and Translation (RST): To remove rigid translation, rotation, and scale variability from observed images, we use a principal component approach. For each image $I$, we define the mean location and a covariance matrix as

$\bar{x}_{I}=\sum_{\mathbb{R}^{2}} x \tilde{I}(x)$, and $K_{I}=\sum_{\mathbb{R}^{2}}\left(x-\bar{x}_{I}\right)\left(x-\bar{x}_{I}\right)^{T} \tilde{I}(x)$

where $\tilde{I}(x)=\left(I(x) / \sum_{\mathbb{R}^{2}} I(x)\right)$. Means and eigenvectors of $K_{I}$ are then used to align the two images. This enables us to focus on estimation of $\phi$ without worrying about errors resulting in this initial alignment. Shown in Fig. 6(d) is an example of this initialization.

Shown in Figs. 6-8 are some results obtained using this multiresolution approach on MRI images of a rat brain growth. Shown in Fig. 6 is the first experimental setup. Panel (a) shows the image $I$, (b) shows $J$, (c) shows their initial difference $\mid I-$ $J$, and (d) shows the initial RST alignment of $I$ to match $J$. Then, a multiresolution approach is used to estimate the optimal $\hat{\phi}$. The results for estimating $\hat{\phi}$ are shown in Fig. 7, where (a) shows $J$, (b) shows $I(\hat{\phi})$, and (c) shows their difference $I(\hat{\phi})-J$. Deformations associated with the resulting $\hat{\phi}$ are shown in several ways - a vector field (d), a deformed lattice (e), and an image of displacement magnitudes (f). Fig. 8 shows another example of this estimation using a different pair of $I$ and $J$.

A quick look at the estimated $\hat{\phi}$ s supports the earlier claim that growth is local and the deformation field can be locally modeled using radial displacements around random seeds. The next section elaborates on this idea. As stated earlier, the solution $\hat{\phi}$ is not unique; it depends on the minimization criterion and the optimization procedure and will affect the remaining analysis.

\section{ESTIMATION OF GRID VARIABLES}

So far, we have estimated the deformation $\hat{\phi}$ that matches the image $I$ to the image $J$. Next, we consider the problem of estimating GRID variables-seed locations, RDFs, and

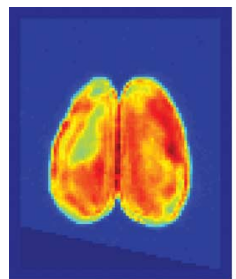

(a)

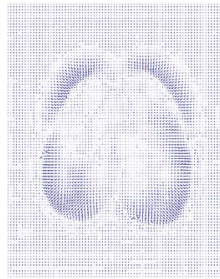

(d) (b)

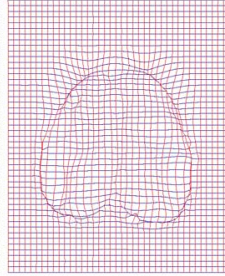

(e)

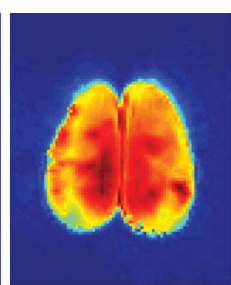

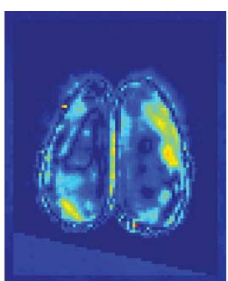

(c)

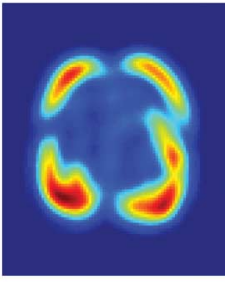

(f)
Fig. 7. (a) $J$, (b) $I(\hat{\phi})$, (c) $I(\hat{\phi})-J$, (d) deformation vector field generating $\hat{\phi}$, (e) optimal $\hat{\phi}$ applied to uniform lattice, and (f) image of $|d \hat{\phi}|$ averaged over small neighborhoods to show active regions.

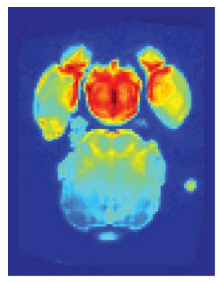

(a)

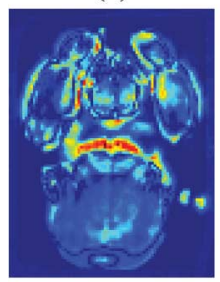

(e)

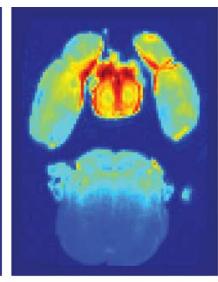

(b)

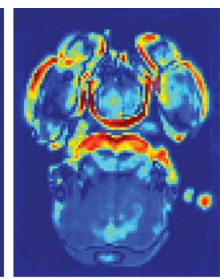

(c)

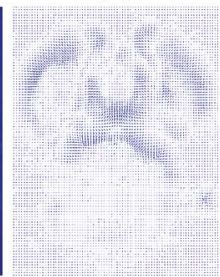

(f)

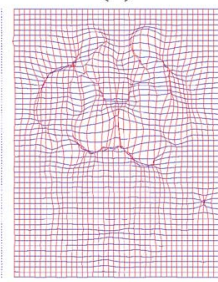

(g)

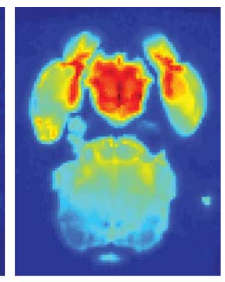

(d)

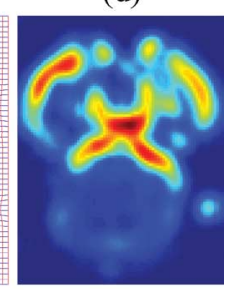

(h)
Fig. 8. Estimated deformation that optimally matched $I$ with $J$ : (a) $I$, (b) $J$, (c) $I$ after RST, (d) $I(\hat{\phi})$, (e) $I(\hat{\phi})-J$, (f) optimal deformation field, (g) optimal $\hat{\phi}$ applied to uniform lattice, and (h) $|d \hat{\phi}|$ averaged over small neighborhoods to show active regions.

ADFs-from the estimated $\hat{\phi}$. Our approach is to treat $\hat{\phi}$ as a noisy observation of the underlying GRID deformation and use a maximum-likelihood setup to estimate GRID variables.

\section{A. Model for Displacement Field}

We will analyze deformations in the form of displacement vector fields and, therefore, we model them explicitly. For a deformation $\hat{\phi}$, the displacement vector at any point $x$ is given by $d \hat{\phi}(x) \equiv \hat{\phi}(x)-x$. First, we derive a model for displacement field induced by the GRID model. An elementary displacement field, attributed to a single seed, is given in the Cartesian coordinates as

$\theta(v ; A, \delta)=R(\|v\| ; \delta) A(\tau(v)) u(\tau)$, where $u(\tau)=\left[\begin{array}{l}\sin (\tau) \\ \cos (\tau)\end{array}\right]$

and $v=x-x_{\text {seed. }}$ As mentioned earlier, $A$ is the ADF that captures the deformation around the seed. It is a function of 
the angle $\tau$ made by the vector $v$ with the positive horizontal axis. To reduce notation, we assume momentarily that the seed is located at the origin, i.e., $x_{\text {seed }}=0$. The deformation model simplifies to

$$
\theta(x ; A, \delta)=R(\|x\| ; \delta) A(\tau(x)) u(\tau) .
$$

$\tau(x)$ is the counterclockwise angle made by the vector $x$ with the positive horizontal direction. Here, $\theta$ can be viewed as a "template displacement" of a uniform lattice, induced by the ADF $A$ and the RDF $R$. For later convenience, we define a vector field $G(x ; \delta)=R(\|x\| ; \delta) u(\tau)$ so that we can rewrite $\theta(x ; A, \delta)=G(x ; \delta) A(\tau(x))$.

\section{B. Observation Model}

We are given a displacement vector field $d \hat{\phi}$ such that for any $x \in \mathbb{R}^{2}, d \hat{\phi}(x)$ is a 2-D vector. Our goal is to estimate GRID variables using the given deformation field. We first impose a data model on the observed deformation field that will allow us a likelihood-based framework for the estimation of unknowns. We assume that the observed deformation is a noisy version of the one dictated by the GRID model, i.e.,

$$
d \hat{\phi}(x)=\sum_{i=1}^{n} \theta\left(x-x_{\text {seed }}^{i} ; A^{i}, \delta_{i}\right)+N(x)
$$

where $N(x) \in \mathbb{R}^{2}$ is a vector of independent Gaussian random variables, with mean zero and a fixed variance $\sigma^{2}$. Each of the $\theta \mathrm{s}$ are as specified in (7). The number of seeds $n$ is unknown and also needs to be estimated from the observed data. According to this model, the observed deformation is a superposition of several template deformations, each having an associated $\mathrm{ADF} A^{i}$, an RDF that depends on parameters $\delta_{i}$, and a seed placement $x_{\text {seed }}^{i}$. Note that (8) is a simplification of the original GRID dynamics, based on the assumption that seeds are placed far enough to have nonoverlapping displacements in the image. That is, $\sum_{x}\left\langle\theta\left(x-x_{\text {seed }}^{i} ; A^{i}, \delta_{i}\right), \theta\left(x-x_{\text {seed }}^{j} ; A^{j}, \delta_{j}\right)\right\rangle=0$ for $i \neq j$. This is motivated by the exponential decay in the influence of a seed as a function of the radial distance. In case these displacements interact, the overall displacements will not be a simple linear combination. We can view this model as a decomposition of a cumulative deformation into simpler, more elementary deformations, each of which belongs to the space

$$
\begin{aligned}
\left\{\phi(x)=x+\theta\left(x-x_{\text {seed }} ; A, \delta\right) \mid x_{\text {seed }}\right. & \in \mathbb{R}^{2}, \\
& \left.A \in \mathbb{L}^{2}\left(\mathbb{S}^{1}\right), \delta \in \mathbb{R}_{+}^{2}\right\} .
\end{aligned}
$$

Note that this space of elementary deformations is not a vector space. This decomposition is different from more traditional component analysis of observations (e.g., principal component analysis or sparse representations of signals) in that the components here are restricted to be predefined elementary deformations, elements of the space given above.

Under this model, the negative of log-likelihood function, also referred to as likelihood energy, is given by

$$
L \equiv \int_{\mathbb{R}^{2}}\left\|d \hat{\phi}(x)-\sum_{i=1}^{n} \theta\left(x-x_{\text {seed }}^{i}, A^{i}, \delta_{i}\right)\right\|^{2} d x
$$

where $\|\cdot\|$ implies the 2-norm of a vector. Let $\Theta$ be the set of all unknowns: $\Theta=\left\{n,\left(x_{\text {seed }}^{i}, A^{i}, \delta_{i}\right), i=1,2, \ldots, n\right\}$. Here, $n$ is a non-negative integer, $x_{\text {seed }}^{i} \in \mathbb{R}^{2}, \delta_{i} \in \mathbb{R}_{+}$or $\mathbb{R}_{+}^{2}$ depending upon the parametric family for RDF, and $A^{i}: \mathbb{S}^{1} \mapsto \mathbb{R}$ is a square-integrable function, i.e., $A^{i} \in \mathbb{L}^{2}\left(\mathbb{S}^{1}\right)$.

\section{Maximum Likelihood Estimation}

Choosing MLE as the framework for variable estimation, we formulate the estimation problem $\hat{\Theta}=\operatorname{argmin}_{\Theta} L(\Theta)$. This solution is equivalent to maximizing the magnitude of the projection of $d \hat{\phi}$ onto the space of GRID deformations. We will estimate $\hat{\Theta}$ using an iterative approach, where the elements of $\Theta$ are updated iteratively. Since this approach is gradient based, it is important to have a good initialization of unknown variables. In this section, we derive and outline steps for updating components of $\Theta$, while the remaining components are held fixed.

For each $i$, define the residual deformation, $d \hat{\phi}_{i}(x)=$ $d \hat{\phi}(x)-\sum_{j \neq i} \theta\left(x-x_{\text {seed }}^{j} ; A^{j}, \delta_{j}\right) . d \hat{\phi}_{i}$ is the residual deformation field in $d \hat{\phi}$ after removing contributions from all seeds except the $i$ th seed. These residual are computed using the current values of GRID variables. To minimize computational cost, we first construct the templates $\theta_{i} \equiv \theta\left(x-x_{\text {seed }}^{i} ; A^{i}, \delta_{i}\right)$, $i=1,2, \ldots, n$. Note that $\theta_{i}$ s are nonzero only on a small subset of $\mathbb{R}^{2}$ around $x_{\text {seed }}^{i}$ and zero on the remaining locations. The GRID variables associated with the $i$ th seed are updated as follows.

1) Update $A^{i}$ : According to

$$
A^{i}(\tau)=\frac{\int_{x \in X_{\tau}}\left\langle d \hat{\phi}_{i}(x), G\left(x-x_{\text {seed }}^{i} ; \delta_{i}\right)\right\rangle d x}{\int_{x \in X_{\tau}}\left\|G\left(x-x_{\text {seed }}^{i} ; \delta_{i}\right)\right\|^{2} d x}
$$

where $X_{\tau}$ denotes the set of all $x$ such that the vector $x-$ $x_{\text {seed }}^{i}$ makes the angle $\tau$ with the positive horizontal axis. The mapping $G$ was introduced in Section V-A

2) Update $x_{\text {seed }}^{i}$ : Since the gradient-based update of $x_{\text {seed }}^{i}$ seems complicated, we resort to a direct estimation of $x_{\text {seed }}^{i}$ as follows:

$$
x_{\text {seed }}^{i}=\underset{\eta}{\operatorname{argmin}} \int_{x}\left\|d \hat{\phi}_{i}(x)-\theta\left(x-\eta ; A^{i}, \delta_{i}\right)\right\|^{2} d x \text {. }
$$

The computation of this minimizer is cheap since the template $\theta\left(x ; A^{i}, \delta_{i}\right)$ needs to be constructed only once. Decomposing the cost function

$$
\begin{aligned}
x_{\text {seed }}^{i} & =\underset{\eta}{\operatorname{argmin}} \int_{x}\left\|d \hat{\phi}_{i}(x)-\theta\left(x-\eta ; A^{i}, \delta_{i}\right)\right\|^{2} d x \\
& =\underset{\eta}{\operatorname{argmin}} \int_{x}\left(\left\|d \hat{\phi}_{i}(x)\right\|^{2}+\left\|\theta\left(x-\eta ; A^{i}, \delta_{i}\right)\right\|^{2}\right. \\
& \left.\quad-2\left\langle d \hat{\phi}_{i}(x), \theta\left(x-\eta ; A^{i}, \delta_{i}\right)\right\rangle\right) d x \\
& \approx \underset{\eta}{\operatorname{argmax}} \int_{x}\left\langle d \hat{\phi}_{i}(x), \theta\left(x-\eta ; A^{i}, \delta_{i}\right)\right\rangle d x .
\end{aligned}
$$

The last step results from assuming that $c_{i}$, contained in $\delta_{i}$, is small enough and $\eta$ lies in the interior of the image domain so that $\int\left\|\theta\left(x-\eta ; A^{i}, \delta_{i}\right)\right\|^{2} d x$ does not change with $\eta$. Defining $\bar{\theta}(x)$ to be $\theta(-x)$, the inner product in 


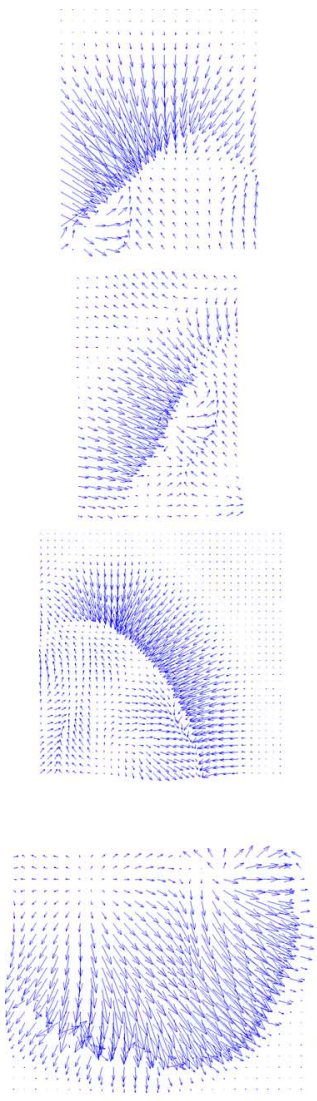

(a)
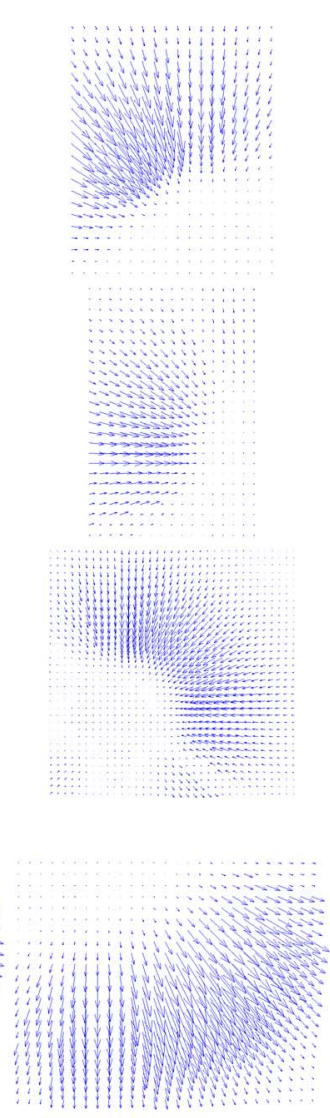

(b)
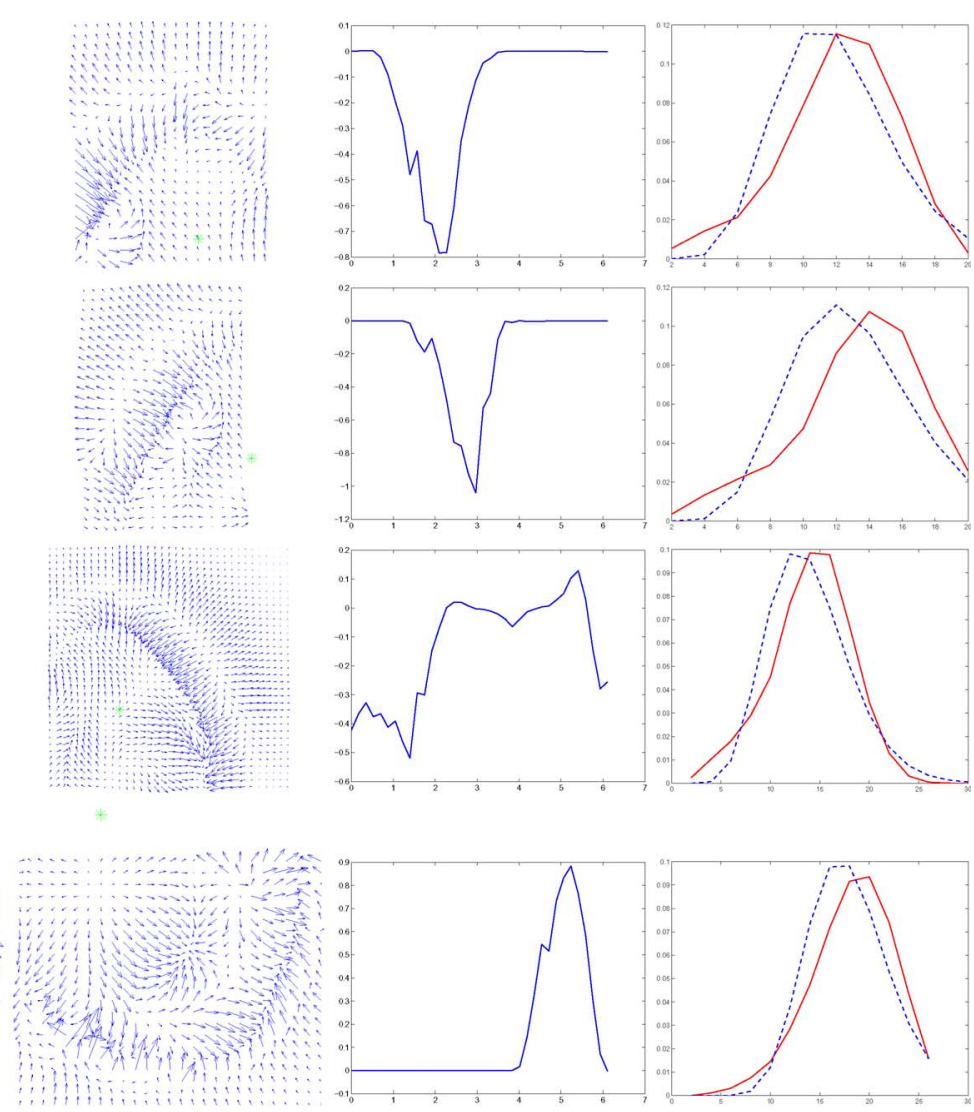

(c)

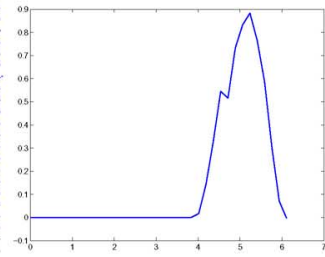

(d)

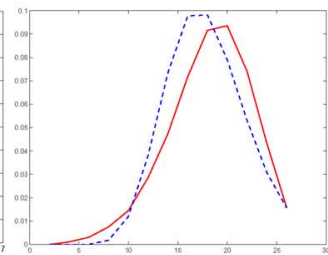

(e)

Fig. 9. MLE of GRID variables for deformation shown in Fig. 7. (a) Original deformation $d \hat{\phi}$, (b) estimated GRID deformation, (c) residual deformation, (d) ADFs, and (e) estimated (solid line) and observed (dotted line) RDFs.

(11) becomes a 2-D convolution between $d \hat{\phi}$ and $\bar{\theta}$. Define the 2-D Fourier transforms

$$
\begin{aligned}
& F_{d}(\omega)=\int_{x} d \hat{\phi}_{i}(x) e^{-j x \omega} d x \\
& F_{\theta}(\omega)=\int_{x} \bar{\theta}\left(x ; A^{i}, \delta_{i}\right) e^{-j x \omega} d x
\end{aligned}
$$

for $j=\sqrt{-1}$. Then, we can obtain the maximizer as $x_{\text {seed }}^{i}=\operatorname{argmax}_{\eta} \int_{\omega} F_{d}(\omega) F_{\theta}(\omega) e^{j \omega \eta} d \omega$. Since the function to be maximized is the inverse Fourier transform of the product $F_{d}(\omega) F_{\theta}(\omega)$, it is fast to compute.

3) Update $\delta_{i}$ : Again, we perform this step using an exhaustive search since a gradient-based update will be computationally expensive. The update is $\delta_{i}=\operatorname{argmin}_{\delta} \int_{x} \| d \hat{\phi}_{i}(x)-$ $\theta\left(x-x_{\text {seed }}^{i} ; A^{i}, \delta\right) \|^{2} d x$.

In this iterative strategy, one has to specify a stopping criterion to the algorithm. Here, the stopping criterion also corresponds to determining the estimated value of $n$, the number of seeds. Since $n$ is unknown beforehand, one can use a penalty function, perhaps a complexity criterion, in addition to the likelihood function. An equivalent strategy is to stop when the contribution from the next seed, measured by the quantity $\| \theta(x-$ $\left.x_{\text {seed }} ; A, \delta\right) \|$, is smaller than a certain threshold. We have used this criterion for stopping the algorithm with a threshold value determined through experimentation.

Shown in Figs. 9 and 10 are results of this estimation procedure on the deformations shown in Figs. 7 and 8, respectively. In each row of these figures, we show estimated contributions from individual seeds in rectangular regions around them. The first picture shows a portion of the original $d \hat{\phi}$, the second shows the estimated $\theta$ function for that seed, and the third shows the residual $d \hat{\phi}-\theta$ in the same region (with the seed location shown by a star). The next two plots show the ADFs and the RDFs associated with that seed placement. The negative and positive values of ADFs denote directional decay and growth, respectively. In some cases, ADF shows a unipolar source or sink, while in other cases we see multipolar behaviors. It can be seen that in cases where $d \hat{\phi}$ has sharp boundaries (e.g., Fig. 9 third row), the GRID model does not explain completely the observed variations. This is due to the smoothness of RDFs and can be addressed by choosing different RDFs. In all cases, the estimated GRID variables seem to capture the general deformation patterns and may prove sufficient for characterizing and classifying the overall growth. Fig. 11 shows the evolutions of the likelihood energy $L$ as successive seeds are detected by the iterative algorithm, for the two datasets. After a stage, addition of another seed does not result in a substantial decrease in $L$ and the algorithm terminates. We can reconstruct the displacement feed attributed to the estimated radial patterns using 


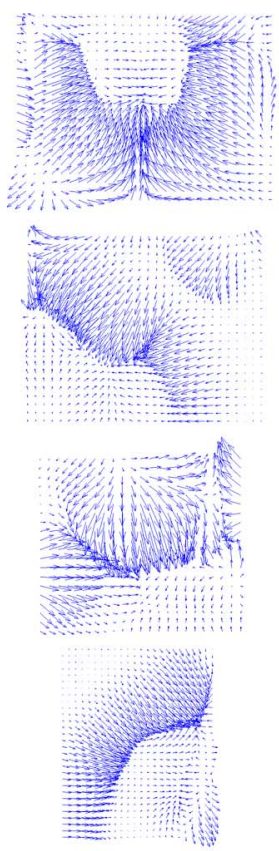

(a)

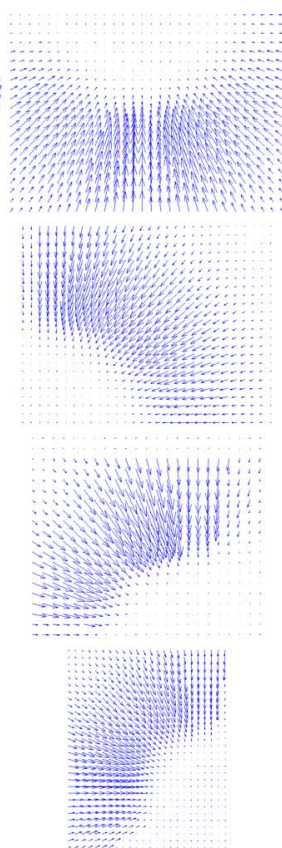

(b)

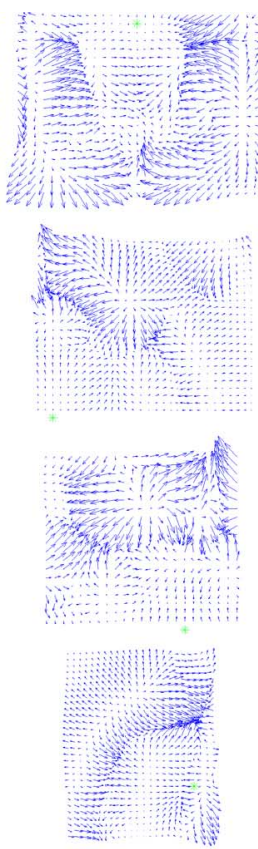

(c)
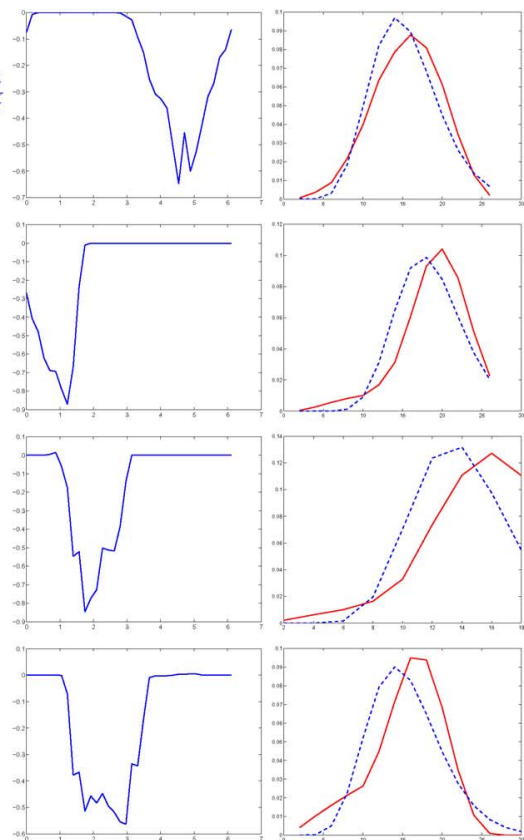

(d)
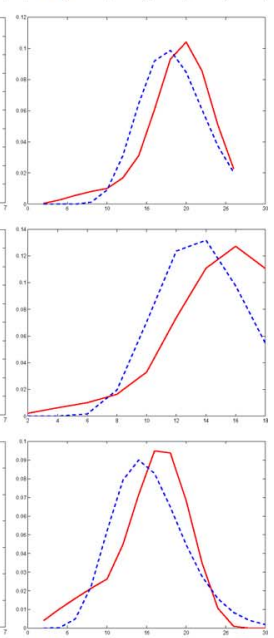

(e)

Fig. 10. MLE of GRID variables for deformation shown in Fig. 8.

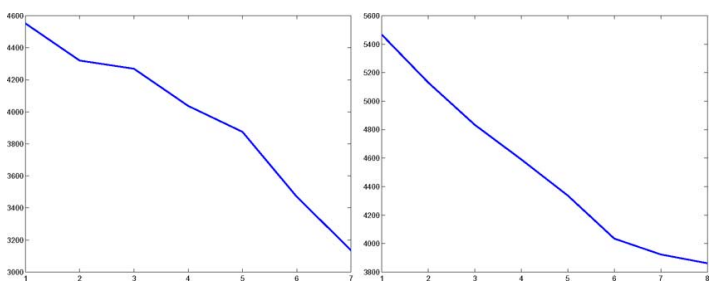

Fig. 11. Evolution of $L$ as seeds are detected sequentially for two datasets, respectively.
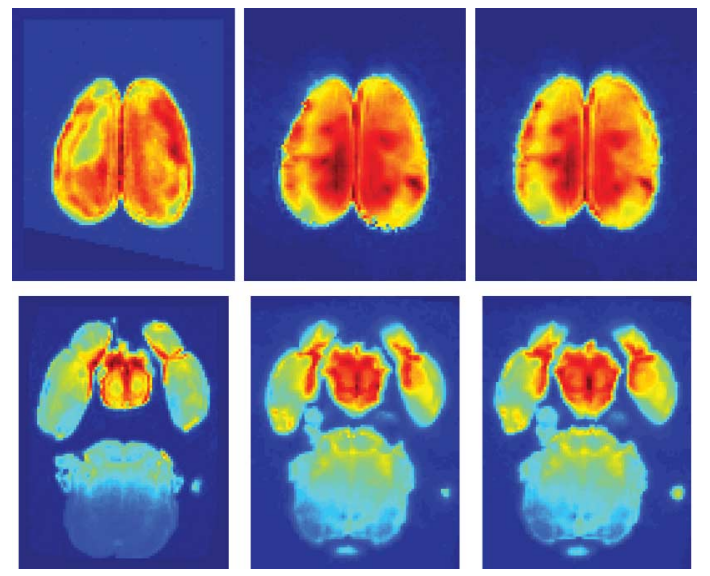

Fig. 12. GRID reconstruction of growth: in each row, left panel is $J$, middle panel is $I(\hat{\phi})$, and right panel is $I(\tilde{\phi})$.

$d \tilde{\phi}(x) \equiv \sum_{i=1}^{n} \theta\left(x-\hat{x}_{\text {seed }}^{i} ; \hat{A}^{i}, \hat{\delta}_{i}\right)$, so that $\tilde{\phi}(x)=x+d \tilde{\phi}(x)$. Shown in Fig. 12 are two examples of $I(\tilde{\phi})$, compared to images $I(\hat{\phi})$ resulting from originally estimated deformations.

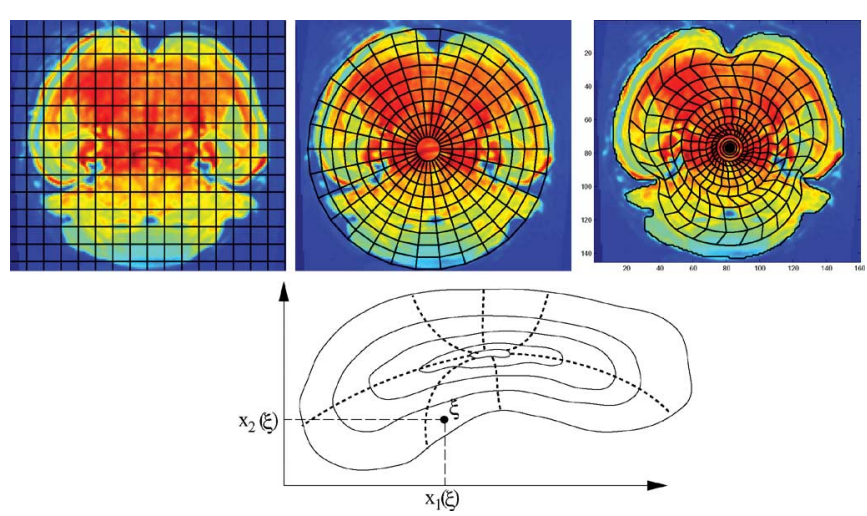

Fig. 13. Examples of different coordinate systems: cartesian (first), polar (second), and curvilinear (third). Bottom diagram shows Darcyan (curvilinear) coordinate system.

\section{Possible ExtEnsions}

Although the model presented so far provides a valuable decomposition of an observed growth in biological plausible elementary units, there are several extensions of this model that can make it even more useful. In this section, we outline some of these ideas.

1) Darcyan Coordinate System: In the problem of growth analysis, it will be useful to perform analysis in a coordinate system that changes with the anatomy. Shown in Fig. 13 are three examples of coordinate systems imposed on an MRI image of a rat brain. Rather than choosing a fixed Cartesian system Fig. 13(a) or a polar system Fig. 13(b), we suggest a time-varying coordinate system, say with the coordinates $\xi=\left(\xi_{1}, \xi_{2}\right) \subset \Xi$. This system is usually curvilinear in $\mathbb{R}^{2}$ as shown in the third and the bottom panels of Fig. 13. The solid lines denote the level sets of variable $\xi_{1}$, while the dotted lines denote 
the level sets of $\xi_{2}$. One can view $\xi$ as a permanent "tag" associated with each particle in the object, while the actual location of that point is given by the function $x$, such that $x(\xi)=\left[x_{1}\left(\xi_{1}, \xi_{2}\right), x_{2}\left(\xi_{1}, \xi_{2}\right)\right] \in X(0)$. As the organism develops, the Darcyan coordinate system too will develop, and the time-varying locations of $\xi$ are given by $x(\xi, t) \in X(t)$. We call this system the Darcyan, after the celebrated author D'Arcy Thompson [23]. All biological decisions during development should be expressed in Darcyan coordinates. The time parameter $t$ measures time from the instant when the initial image is taken.

2) Parametric Forms of ADF: So far, we have used a nonparametric form for the ADFs, but it may be possible to use a mixture of von Mises density functions to make it a fully parametric model. The von Mises density function on $\mathbb{S}^{1}$ is given by $f(\tau)=\left(1 / I_{0}(\kappa)\right) \exp \left(\kappa \cos \left(\tau-\tau_{0}\right)\right)$, where $\tau_{0}$ denotes the mean value, $\kappa$ is a measure of variance, and $I_{0}$ is a modified Bessel function that normalizes the density function. This density can model unipolar seeds, but for multipolar seeds we suggest a mixture of von Mises variables.

3) Catalog of Growth and MAP Estimation: Perhaps the most significant future work in this approach is to develop a probabilistic catalog of ADFs using annotated datasets. One would like to associate certain types of deformations, represented by their corresponding ADFs, with specific growth conditions. For example, in case of later stages of a malignant tumor what ADFs can model the growths with high probability? Such probabilistic catalogs will have multiple uses: they can then be used as prior models for MAP estimation of GRID variables or they can be used to classify a particular growth into predetermined classes.

4) Direct Estimation of GRID Variables: In this paper, we have used a two-step procedure to estimate GRID variables. A more desirable approach will be to directly estimate GRID variables from the image data. We sketch an approach for estimating the Poison intensity measure $F$ underlying the placement of seeds. Say we want to solve the minimization problem: $\hat{F}=\operatorname{argmin}_{F \in \mathcal{F}} \int_{\mathbb{R}^{2}}(I(x(\xi, 0))-$ $J(x(\xi, T)))^{2} d x$, where

$x(\xi, T)=x(\xi, 0)+\sum_{t=1}^{T}\left(\int_{\eta} \theta(x(\xi, t)-x(\eta, t)(F(d \eta))\right.$.

A measure $F$ is an extremum of the cost function if it satisfies the condition

$$
\begin{aligned}
\int_{\xi} \xi_{1}(I(z(\xi, T))-J(x(\xi, 0)) \times \\
\quad\left(\left\langle\nabla I(z(\xi, T)),\left(\sum_{t=1}^{T} \theta(x(\xi, t)-x(\kappa, t))\right)\right\rangle\right) d \xi=0
\end{aligned}
$$

for all $\kappa$ where $z(\xi, T)=x(\xi, 0)+\sum_{t=1}^{T} \int_{\eta}$ $\theta(x(\xi, t)-x(\eta, t)) F(d \eta)$. To understand this condition, consider the term $\sum_{t=1}^{T} \theta(x(\xi, t)-x(\kappa, t))$. This is the total resulting displacement vector of the particle $\xi$, over the interval $[0, T]$, if a seed is placed at $\kappa \in \Xi$. As a function of $\xi$, this term defines a vector field on the set $\Xi . z(\xi, T)$ is the expected new location of the particle $\xi$ at time $T$, the expectation is with respect to the Poisson measure $F$. $\nabla I$ is the gradient of image $I$ evaluated on a background space that has been deformed from $x(\xi, 0)$ to $z(\xi, T)$. The term $K(\xi) \equiv\left\langle\nabla I(z(\xi, T)),\left(\sum_{t=1}^{T} \theta(x(\xi, t)-x(\kappa, t))\right)\right\rangle$ denotes the inner product of these two vector fields and defines a scalar field on $\Xi$. The extremum condition stated above says that the inner product of the scalar field $(I(z(\xi, T))-J(x(\xi, 0))$ and $K(\xi)$ is zero for all $\kappa$.

5) Statistical Analysis of Growth: An important application of this framework is in analyzing observed growth for classification into normal and abnormal growth. Using statistics of the deformations, it should be possible to efficiently classify local growth patterns. In this paper, we have developed efficient representations of anatomical growth and statistical analysis of these quantities under different classes should lead to tools for growth classification. Another important area of research can be the use of ideas from functional analysis of variance (FANOVA) for studying effects of drugs and treatments on anatomical growth, when growth is represented using the GRID variables.

6) 3-D Growth Models: The discussion in this paper is mostly restricted to 2-D image data, while the study of biological growth is more natural in 3-D spaces. In principle, except for increased computational cost, it should be straightforward to extend the GRID framework to 3-D growth analysis. An important change is going from 2-D to 3-D will be that the angular deformation function ADF will now be from $\mathbb{S}^{2}$ to $\mathbb{R}$, and one will need some parametric families to make this representation efficient.

\section{SUMMARY}

We have proposed a mathematical framework for modeling growth of biological objects, such as anatomical parts, with a focus on locally active growth. This GRID model uses random placements of seeds and radial deformations around those seeds to capture growth-based deformations. The resulting growth patterns can be characterized using these GRID variables. We have demonstrated MLE of GRID variables using MRI images of a rat's brain growth. This paper accomplishes the following tasks.

1) It introduces a mathematical representation of deformation (resulting from anatomical growth) that seems natural for growth analysis.

2) It presents some useful analytical properties (Jacobian, divergence, curl, etc.) of these deformations that can be useful in growth analysis and anatomical interpretations.

3) It presents potential probability models for variables modeling GRID deformations, for future Bayesian inferences.

4) It outlines possible extensions of this framework to handle more practical situations and to eliminate some of its shortcomings.

\section{ACKNOWLEDGMENT}

The authors would like to thank Dr. J. Zhang of the Johns Hopkins University for providing them with the MRI data used 
in this paper. The authors gratefully acknowledge several useful remarks by the three anonymous reviewers.

\section{REFERENCES}

[1] J. A. Sherratt and M. A. Chaplain, "A new mathematical models for avascular tumour growth," J. Math. Biol., vol. 43, no. 4, pp. 291-312, 2001.

[2] I. L. Dryden and K. V. Mardia, Statistical Shape Analysis. New York: Wiley, 1998

[3] F. Bookstein, The Measurement of Biological Shape and Shape Changes, ser. Lecture Notes Biomathematics. New York: Springer Verlag, 1978, vol. 24.

[4] D. G. Kendall, D. Barden, T. K. Carne, and H. Le, Shape and Shape Theory. New York: Wiley, 1999.

[5] U. Grenander and M. I. Miller, "Computational anatomy: An emerging discipline," Quart. Appl. Math., vol. LVI, no. 4, pp. 617-694, 1998.

[6] M. I. Miller and L. Younes, "Group actions, homeomorphisms, and matching: A general framework," Int. J. Comput. Vision, vol. 41, no. 1/2, pp. 61-84, 2002.

[7] A. Trouve, "Diffemorphisms groups and pattern matching in image analysis," Int. J. Comput. Vision, vol. 28, no. 3, pp. 213-221, 1998.

[8] P. M. Thompson and A. W. Toga, "A framework for computational anatomy," Computing Visualization Sci., vol. 5, pp. 13-34, 2002.

[9] M. Miller, G. Christensen, Y. Amit, and U. Grenander, "Mathematical textbook of deformable neuroanatomies," in Proc. Nat. Acad. Sci., Dec. 1993, vol. 90, no. 24.

[10] V. Arsigny, X. Pennec, and N. Ayache, Polyrigid and Polyaffine Transformations: A New Class of Diffeomorphisms for Locally Rigid or Affine Registration, ser. Lecture Notes Computer Science, vol. 2879 , R. E. Ellis and T. M. Peters, Eds. New York: Springer, 2003, pp. 829-837.

[11] P. R. Andresen and M. Nielsen, "Non-rigid registration by geometryconstrained diffusion," in Proc. MICCAI, 1999, vol. 1679, pp. 533-543.
[12] V. G. Kac, Infinite-Dimensional Lie Algebras, 3rd ed. Cambridge, MA: Cambridge Univ. Press, 1990.

[13] O. Clatz, P.-Y. Bondiau, H. Delingette, G. Malandain, M. Sermesant, S. K. Warfield, and N. Ayache, "In silico tumor growth: Application to glioblastomas," in Proc. MICCAI 2004, LNCS 3217, C. Barillot, D. Haynor, and P. Hellier, Eds., 2004, p. 337345.

[14] A. R. Kansal, S. Torquato, G. R. H. , IV, E. A. Chioccaeb, and T. S. Deisboeck, "Simulated brain tumor growth dynamics using a threedimensional cellular automaton," J. Theor. Biol., vol. 203, pp. 367-382, 2000.

[15] K. R. Swanson, C. Bridge, J. Murray, and E. C. A. , Jr., "Virtual and real brain tumors: Using mathematical modeling to quantify glioma growth and invasion," J. Neurological Sci., vol. 216, pp. 1-10, 2003.

[16] M. A. J. Chaplain, Mathematical Modeling of Tumor Growth, ser. Series: Interdisciplinary Applied Mathematics. New York: Springer, 2006.

[17] Y. Ling and B. He, "Entropic analysis of biological growth models," IEEE Trans. Biomed. Eng., vol. 40, no. 12, pp. 1193-1200, Dec. 1993.

[18] W. Harvey, Essays on Generation of Animals 1651.

[19] G. E. Christensen, R. D. Rabbitt, and M. Miller, "A deformable neuroanatomy textbook based on viscous fluid mechanics," in Proc. 27th Annu. Conf. Information Sciences Systems, J. Prince and T. Runolfsson, Eds., Baltimore, MD, Mar. 24-26, 1993, pp. 211-216, Dept. Electrical Engineering, The Johns Hopkins Univ.

[20] B. Kim, J. L. Boes, K. A. Frey, and C. R. Meyer, "Mutual information for automated unwarping of rat brain autoradiographs," Neuroimage, vol. 5, no. 1, pp. 31-40, 1997.

[21] F. L. Bookstein, "Size and shape spaces for landmark data in two dimensions," Stat. Sci., vol. 1, pp. 181-242, 1986.

[22] N. Khaneja, M. Miller, and U. Grenander, "Dynamic programming generation of curves on brain surfaces," IEEE Trans. Pattern Anal. Mach. Intell., vol. 20, no. 11, pp. 1260-1264, Nov. 1998.

[23] D. W. Thompson, On Growth and Form: The Complete Revised Edition. New York: Dover, 1992. 